\title{
Top quark pair properties - spin correlations, charge asymmetry and complex final states
}

\author{
James William HOWARTH* \\ On behalf of the ATLAS collaboration \\ (University of Manchester (GB)) \\ E-mail: james.william.howarth@cern.ch
}

\begin{abstract}
Results are presented on the observation of non-vanishing spin correlations in $t \bar{t}$ pairs at ATLAS, the measurement of their intrinsic polarisation and charge asymmetry. Comparisons are made between previous results from the Tevatron and current results from the LHC experiments. A new result is presented on the cross section of top pair production in association with heavy flavour quarks with a view to investigating this process as an irreducible background to $t \bar{t} \mathrm{H}$ production. All results presented are consistent with the standard model expectations.
\end{abstract}

XXI International Workshop on Deep-Inelastic Scattering and Related Subjects 22-26 April, 2013

Marseilles, France

\footnotetext{
* Speaker.
} 


\section{Top quark properties}

The top quark is a truly unique particle. With a mass of $173.2 \mathrm{GeV}$ [1] it is the heaviest particle in the standard model (SM) and its mass is such that it decays before it can hadronise and thus one has the opportunity of studying a "naked" quark. Due to its high mass the top quark may play a special role in electroweak symmetry breaking. In the SM top quarks decay exclusively to $\mathrm{W}$ bosons and b-quarks and provide striking signatures in particle detectors. With appropriate selection cuts it is possible to select an almost background free sample of events where both W's decay leptonically, called the "dilepton" channel, and achieve excellent background rejection in events where one of the W's decays leptonically, called the "single lepton" channel. Angular distributions of top quarks and their decay particles can provide detailed information on their spin structure and polarisation, as well as being sensitive to asymmetries in top pair production.

\section{Spin correlations and polarisation}

Spin correlation and polarisation are closely related, as illustrated in the double differential equation for $t \bar{t}$ decay shown in equation 2.1 [2], where the polarisation of the top quark is denoted by the parameter $\mathbf{P}$ and spin correlation is denoted by the parameter $\mathbf{C}$

$$
\frac{1}{\sigma} \frac{d^{2} \sigma}{d\left[\cos \left(\theta_{i}\right)\right] d\left[\cos \left(\theta_{j}\right)\right]}=\frac{1}{4}\left[1+\mathbf{P} \alpha_{i} \cos \left(\theta_{i}\right)+\mathbf{P} \alpha_{j} \cos \left(\theta_{j}\right)+\mathbf{A} \alpha_{i} \alpha_{j} \cos \left(\theta_{i}\right) \cos \left(\theta_{j}\right)\right] .
$$

The spin information of the top quark may be accessed by its decay particles. Each decay particle in the top decay chain carries this spin information to a different degree. This is represented in equation 2.1 by $\alpha_{i / j}$. Charged leptons from $\mathrm{W}$ decays carry the full spin information of the parent top quark at leading order ( $\mathrm{LO}) \alpha_{\ell}=1.0$ [2]. The degree of spin correlation is predicted by the SM and since QCD is parity invariant, top quarks are predicted to have no intrinsic polarisation arising from QCD. Both hypotheses have been tested in ATLAS [3] $7 \mathrm{TeV}$ data.

\subsection{Spin correlation results}

In order to maximise the sensitivity to spin correlations, a dilepton selection was used. Both $\alpha_{i / j}$ parameters are equal to 1 for charged leptons, allowing access to the full spin information of the parent top quarks in the dilepton channel. Due to the two neutrinos in the final state, the kinematics of dilepton events are under-constrained and reconstruction is difficult. Fortunately the difference in the azimuthal angle between the charged leptons in the laboratory frame $(\Delta \phi)$ is sensitive to spin correlations and no reconstruction is necessary. Dilepton events were selected from $2.1 \mathrm{fb}^{-1}$ of ATLAS $7 \mathrm{TeV}$ data with two opposite sign isolated leptons and at least two good quality jets. The selection is identical to previous cross section results [4]. The MC@NLO event generator [5] was used to generate $t \bar{t}$ and single top events, whereas ALPGEN [6] was used to simulate diboson and Drell-Yan events. Background arising from experimentally mis-measured leptons were estimated using a data driven method $[7,8]$ and the normalisation of $Z / \gamma^{*} \rightarrow e^{+} e^{-}, \mu^{+} \mu^{-}$was taken from a control region in data.

A template fit was performed to the $\Delta \phi$ variable in data using two hypotheses templates. One hypothesis template was generated using SM spin correlations and another with no included spin 
correlations. The fit was performed in the $e^{+} e^{-}, \mu^{+} \mu^{-}$and $e^{ \pm} \mu^{\mp}$ simultaneously and the degree to which the data agreed with the SM template was measured $\left(f_{S M}\right)$. In data we observe $f_{S M}=1.30$ \pm 0.14 (stat.) ${ }_{-0.22}^{+0.27}$ (syst.) compared to the SM prediction of $f_{S M}=1$ and the uncorrelated spin hypothesis of $f_{S M}=0$. This result has a significance of 5.1 standard deviations $(\sigma)$ when compared to the uncorrelated spin hypothesis. This was the first observation of non-vanishing spin correlation consistent with a spin $1 / 2$ particle [7].
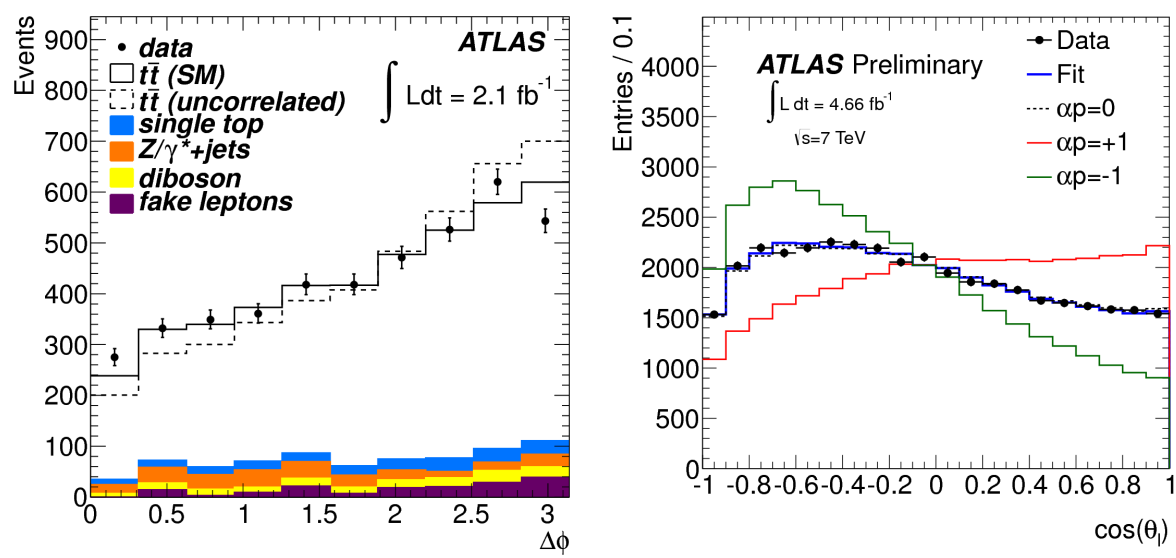

Figure 1: The distribution of $\Delta \phi$ between the two charged leptons in the lab frame in the summed dilepton channels [7] (left) and the fit of the $\cos \theta$ variable in the single muon channel (right) [9].

\subsection{Polarisation results}

Semi-leptonic events were selected using $4.66 \mathrm{fb}^{-1}$ of ATLAS $7 \mathrm{TeV}$ data with exactly one isolated electron or muon and at least four jets, one of which must be b-tagged. The selection used was identical to that used in a previous cross section result [10]. To reconstruct the final state $t \bar{t}$ four vectors, a topological likelihood fitter was used, described in reference [11].

The degree of polarisation was extracted by a template fit to the $\cos \theta$ variable where $\theta$ is the angle between the charged lepton and the helicity of the parent top quark in the top quark rest frame. Templates were generated for maximal polarisation $\alpha P=+1$ and maximal anti-polarisation $\alpha P=-1$. The same template fitting technique was used as in the spin correlation result and the polarisation was found to be $\alpha \mathrm{P}=-0.060 \pm 0.018$ (stat) ${ }_{-0.064}^{+0.046}$ (syst.) [9] consistent with the SM expectation of $\alpha P \sim 0$.

\section{Charge asymmetries}

Top anti-top production at hadron colliders is predicted to be charge symmetric at LO in both gluon-gluon fusion produced $t \bar{t}$ pairs and those created via $q \bar{q}$ annihilation. At next to leading order (NLO) interference effects produce a charge asymmetry in the $t \bar{t}$ pairs produced by $q \bar{q}$ annihilation. Gluon-gluon fusion is charge symmetric to all orders. In a proton anti-proton collider the charge asymmetry manifests itself as the $t$ 's being boosted in the direction of the incoming $q$ 's, and the $\bar{t}$ 's being boosted in the direction of the incoming $\bar{q}$ 's. At a charge symmetric collider the situation is more complicated. The top quark still gain a boost in the direction of one of the incoming proton 
beams. However the anti-quark in the $t \bar{t}$ production cannot be a valence quark but instead must be a sea quark, usually with low X. The result is that the anti-top quark is produced more centrally and the top quark is produced more forward.

Charge asymmetries at NLO are predicted to be small in the SM. However measurements at the Tevatron from CDF and D0 have indicated an excesses on the order of $2 \sigma$ above the SM expectation, particularly in the high $t \bar{t}$ invariant mass region $[12,13]$, making the investigation of this effect a priority at the LHC. Charge asymmetries can be measured by the ratio of the rapidity difference of the top quarks themselves $\left(A_{C}^{t \bar{t}}\right)$ or by their decay leptons in the dilepton case $\left(A_{C}^{\ell \ell}\right)$, illustrated in equation 3.1:

$$
A_{C}^{i j}=\frac{N\left\{\left(\left|y^{i}\right|-\left|y^{j}\right|\right)>0\right\}-N\left\{\left(\left|y^{i}\right|-\left|y^{j}\right|\right)<0\right\}}{N\left\{\left(\left|y^{i}\right|-\left|y^{j}\right|\right)>0\right\}+N\left\{\left(\left|y^{i}\right|-\left|y^{j}\right|\right)<0\right\}}, \quad \text { where } i j=t \bar{t} \text { or } \ell^{+} \ell^{-} .
$$

\subsection{Dilepton channel}

Dilepton events were selected from $4.7 \mathrm{fb}^{-1}$ of ATLAS $7 \mathrm{TeV}$ data using the same selection as was described for the spin correlation analysis in section 2.1. The $t \bar{t}$ final state was reconstructed using a matrix element weighting method to reconstruct top and anti-top quark four momenta [8].

The measured asymmetry was corrected for detector effects and event selection bias using a calibration curve that was derived by simulating different values for asymmetry using MC and performing toy experiments. Three dilepton channel results were combined using the BLUE method $[14,15]$. The lepton asymmetry was measured to be $A_{C}^{\ell \ell}=0.023 \pm 0.012$ (stat.) \pm 0.008 (syst.) compared to a SM expectation calculated using MC@ NLO of $A_{C}^{\ell \ell}=0.004 \pm 0.001$. The top asymmetry was measured to be $A_{C}^{t \bar{t}}=0.057 \pm 0.024$ (stat.) \pm 0.015 (syst.) compared to a SM expectation of $A_{C}^{t \bar{T}}=0.006 \pm 0.002$ [8].

\subsection{Single lepton channel}
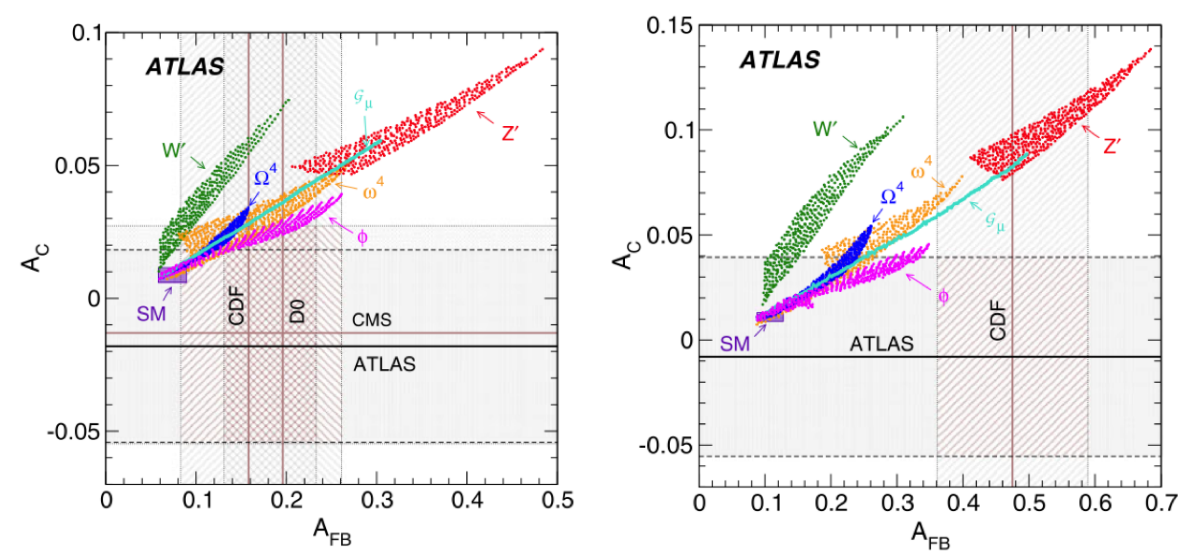

Figure 2: ATLAS single lepton asymmetries result compared to CMS[16], CDF[12] and D0[13] results in the inclusive $m_{t \bar{t}}$ region (left) and high $m_{t \bar{t}}$ region (right). MC predictions are taken from $[17,18]$.

Semi-leptonic events were selected using $1.04 \mathrm{fb}^{-1}$ of ATLAS $7 \mathrm{TeV}$ data with the same selection as that used in the polarisation result described in section 2.2 and reference [19]. The measured rapidity distributions for the top and anti-tops were unfolded to parton level after background subtraction using a Bayesian iterative unfolding procedure using response matrices derived 
from MC@NLO [20]. The unfolding was performed in both an inclusive selection and a two dimensional plane $\left(|y|_{t \bar{t}}, m_{t \bar{t}}\right)$ with two bins in $m_{t \bar{t}}$ split at $450 \mathrm{GeV}$ to coincide with the binning used by CDF [12].

The inclusive charge asymmetry was measured to be $A_{C}^{t \bar{t}}=-0.019 \pm 0.028$ (stat.) \pm 0.024 (syst.) and the differential results were measured to be $A_{C}^{t \bar{t}}=-0.052 \pm 0.070$ (stat.) \pm 0.054 (syst.) for $m_{t \bar{t}}<450 \mathrm{GeV}$ and $A_{C}^{t \bar{t}}=-0.008 \pm 0.035$ (stat.) \pm 0.032 (syst.) for $m_{t \bar{t}}>450 \mathrm{GeV}$. All results are in agreement with the SM expectation of $A_{C}^{t \bar{t}}=0.006 \pm 0.002$. In addition this measurement was compared to results available at the time of publication from CDF and D0 as well as several BSM physics models [17, 18], illustrated in Fig. 2.

This result was combined with the previous dilepton based asymmetry using the BLUE method, resulting in a measurement of $A_{C}^{t \bar{t}}=0.029 \pm 0.018$ (stat.) \pm 0.014 (syst.) with a p-value of 9\% [19].

\section{Heavy flavour final states}
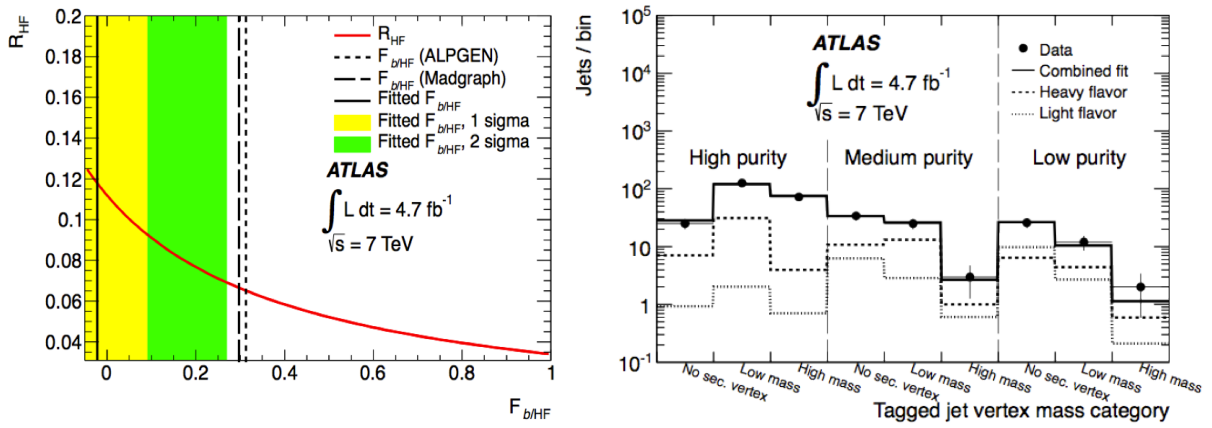

Figure 3: Fitted $R_{H F}$ cross section (left) and template fit as a function of vertex mass (right) [21].

With the discovery of a $125 \mathrm{GeV}$ Higgs boson the measurement of the Higgs-top Yukawa coupling is a priority. Since the decay mode of a $125 \mathrm{GeV}$ Higgs is predominantly via the decay to two b-quarks $(H \rightarrow b \bar{b})$ an understanding of the irreducible backgrounds will be crucial in the measurement of the $t \bar{t} H$ process. One of these backgrounds is the production of $t \bar{t}$ pairs in addition to heavy flavour (HF) where in this case HF is defined as $b \bar{b}, c \bar{c}, b, c$. Production of $t \bar{t}+b \bar{b}, c \bar{c}$ occurs through initial state or final state gluon splitting, $t \bar{t}+b, c$ occurs due to the heavy flavour content of the proton. In addition many BSM models predict additional HF content or events with two $t \bar{t}$ pairs, making this analysis useful for background suppression in future analyses. The measurement was extracted as ratio of fiducial cross sections:

$$
R_{H F}=\frac{\sigma_{f i g}(t \bar{t}+H F)}{\sigma_{f i g}(t \bar{t}+j)}, \quad \sigma_{f i g}(t \bar{t}+H F)=\frac{N_{H F}}{\int \mathscr{L} d t \cdot \varepsilon},
$$

where $\sigma_{f i g}(t \bar{t}+j)$ is the cross section of $t \bar{t}$ events with at least three jets, at least two of which are b-tagged and $\sigma_{f i g}(t \bar{t}+H F)$ is the cross section of $t \bar{t}$ events with three or more jets of which three or more are b-tagged. $N_{H F}$ is the number of jets tagged as having heavy flavour (b or c) in addition to the two b-jets from the top decay and $\varepsilon$ is an acceptance factor derived from ALPGEN MC to convert the number of b-tags from HF jets into the number of events in the signal fiducial region. $\int \mathscr{L}$ is the integrated luminosity of the data. 
Events were selected with the same single lepton selection described in section 3.1 with the exception that the number of b-tags is explicitly two or more for $t \bar{t}+\mathrm{j}$ or three or more for $t \bar{t}+\mathrm{HF}$. The b-tagging algorithm used was neural net based using impact parameter and vertex information with an efficiency of $75 \%$ for b-jets and 35\% for c-jets and a light flavour rejection factor of $\sim 35$ in simulated $t \bar{t}$ events.

The cross section was calculated using a template fit to vertex mass to be $\sigma_{f i g}(t \bar{t}+H F)=0.18$ \pm 0.03 (stat.) $\mathrm{pb}$, compared to the predicted value from ALPGEN of $0.10 \mathrm{pb}$. The $\sigma_{f i g}(t \bar{t}+j)$ is calculated to be $2.55 \pm 0.07$ (stat.) pb compared to the prediction from ALPGEN of $2.83 \mathrm{pb}$. The ratio of cross sections was extracted as $R_{H F}=\left[7.1 \pm 1.3\right.$ (stat.) ${ }_{-2.0}^{+5.3}$ (syst.)]\% [21] compared to the NLO prediction from POWHEG of $[5.2 \pm 1.7] \%$.

\section{Conclusions}

The high statistics of top quark data at the LHC has resulted in measurements of impressive accuracy. Subtle analyses of intrinsic properties and production mechanisms are now possible. ATLAS has produced results on the first observation of non-vanishing top quark spin correlations, intrinsic polarisation, charge asymmetry and associated heavy flavour production.

\section{Acknowledgements}

I would like to thank the following people for their assistance: the ATLAS analysers for providing excellent source material, the organisers of DIS 2013 for a fantastic conference, and Professor Peters for her assistance with compiling these proceedings.

\section{References}

[1] CDF [Tevatron Electroweak Working Group and D0 Collaborations], [arXiv:1305.3929 [hep-ex]].

[2] W. Bernreuther, A. Brandenburg, Z. G. Si and P. Uwer, Phys. Rev. Lett. 87 (2001) 242002 [hep-ph/0107086].

[3] ATLAS Collaboration, JINST 3 (2008) S08003.

[4] ATLAS Collaboration, JHEP 1205 (2012) 059 [arXiv:1202.4892 [hep-ex]].

[5] S. Frixione, F. Stoeckli, P. Torrielli, B. R. Webber and C. D. White, [arXiv:1010.0819 [hep-ph]].

[6] M. L. Mangano, M. Moretti, F. Piccinini, R. Pittau and A. D. Polosa, JHEP 0307 (2003) 001 [hep-ph/0206293].

[7] ATLAS Collaboration, Phys. Rev. Lett. 108 (2012) 212001 [arXiv:1203.4081 [hep-ex]].

[8] ATLAS Collaboration, ATLAS-CONF-2012-057, https://cds.cern.ch/record/1453785.

[9] ATLAS Collaboration, ATLAS-CONF-2012-133, http://cds.cern.ch/record/1478373.

[10] ATLAS Collaboration, ATLAS-CONF-2011-121, http://cds.cern.ch/record/1376413.

[11] ATLAS Collaboration, ATLAS-CONF-2011-033, http://cds.cern.ch/record/1337783.

[12] T. Aaltonen et al. [CDF Collaboration], Phys. Rev. D 83 (2011) 112003 [arXiv:1101.0034 [hep-ex]].

[13] V. M. Abazov et al. [D0 Collaboration], Phys. Rev. D 84 (2011) 112005 [arXiv:1107.4995 [hep-ex]].

[14] L. Lyons et al., Nucl. Instrum. Meth. A 270 (1988) 110.

[15] A. Valassi, Nucl. Instrum. Meth. A 500 (2003) 391.

[16] S. Chatrchyan et al. [CMS Collaboration], Phys. Lett. B 709 (2012) 28 [arXiv:1112.5100 [hep-ex]].

[17] J. A. Aguilar-Saavedra and M. Perez-Victoria, Phys. Rev. D 84 (2011) 115013 [arXiv:1105.4606 [hep-ph]]. 
[18] J. A. Aguilar-Saavedra and M. Perez-Victoria, JHEP 1109 (2011) 097 [arXiv:1107.0841 [hep-ph]].

[19] ATLAS Collaboration, Eur. Phys. J. C 72 (2012) 2039 [arXiv:1203.4211 [hep-ex]].

[20] G. D’Agostini, Nucl. Instrum. Meth. A 362 (1995) 487.

[21] ATLAS Collaboration, [arXiv:1304.6386 [hep-ex]]. 